\title{
The intersection of the compatible linear extensions of a natural partial order
}

\author{
Szilvia Szilágyi
}




\title{
THE INTERSECTION OF THE COMPATIBLE LINEAR EXTENSIONS OF A NATURAL PARTIAL ORDER
}

\author{
SZILVIA SZILÁGYI
}

Received 16 January, 2012

\begin{abstract}
For an acyclic function $f: A \longrightarrow A$ we define a natural $f$-compatible partial order $\hat{f}$ on $A$ and determine the intersection of the $f$-compatible linear extensions of $\hat{f}$.

2000 Mathematics Subject Classification: 06A06; 06F99.

Keywords: compatible partial order, compatible linear extension
\end{abstract}

\section{INTRODUCTION}

One of the central problems in the theory of ordered algebras is to find necessary and sufficient conditions for the existence of a compatible linear extension of $r$ in a partially ordered algebraic structure $\left(A, F, \leq_{r}\right)$. If $F=\varnothing$, then Szpilrajn proved that any partial order $r\left(\leq_{r}\right)$ on a set $A$ can always be extended to a linear order $R$ and any partial order is the intersection of its linear extensions (see [5]). In the present paper we consider a partial order $r$ on the set $A$ and an order endomorphism $f: A \longrightarrow A$ with the natural compatibility property: $x \leq_{r} y$ implies $f(x) \leq_{r} f(y)$ for all $x, y \in A$. Clearly, the pair $(A, f)$ is a unary algebra and the above $f$-compatibility condition allows us to view the triple $\left(A, f, \leq_{r}\right)$ as a partially ordered unary algebra. For $F=\{f\}(f: A \rightarrow A$ is a unary operation) the above mentioned extension problem has been thoroughly investigated. Szigeti and Nagy proved that the partial order $r$ of a unary algebra $\left(A, f, \leq_{r}\right)$ has an $f$-compatible linear extension if and only if the function $f: A \longrightarrow A$ is acyclic (see [3]). For an acyclic $\left(A, f, \leq_{r}\right)$ the intersection of the $f$-compatible linear extensions of $r$ is determined in [2]. For an arbitrary $\left(A, f, \leq_{r}\right)$ the maximal $f$-compatible partial order extensions of $r$ and the intersection are investigated in [1] and [4]. The aim of the present paper is to determine the intersection of the $f$-compatible linear extensions of $\hat{f}$, where the $f$-compatible partial order $\hat{f}$ on $A$ can be defined in a natural way starting from an acyclic function $f: A \longrightarrow A$. 


\section{Preliminaries}

Let $(A, f)$ be a (mono-)unary algebra. A partial order $r$ on $A$ is called $f$-compatible if the function (unary operation) $f: A \longrightarrow A$ is an order endomorphism with respect to $r$. We shall make use of the following notation

$\mathscr{L}\left(A, f, \leq_{r}\right)=\{R \mid r \subseteq R \subseteq A \times A$ is an $f$-compatible linear order on $A\}$.

The intersection

$$
r_{f}=\operatorname{cl}\left(A, f, \leq_{r}\right)=\bigcap_{R \in \mathscr{L}(A, f, \leq r)} R
$$

is called the closure of $r$ with respect to $f$. Indeed, the above definition gives a closure operator (with the monotone, idempotent and extensive properties) on the set of the $f$-compatible partial orders of $A$.

Definition 1 (see [3]). Let $A \neq \varnothing$ be a set and $N \geq 0$ be an integer. A function $f: A \rightarrow A$ takes $N$ steps on the element $x \in A$, if

$$
x, f(x), f^{2}(x), \ldots, f^{N}(x)
$$

are different elements in $A$, and

$$
f^{N+1}(x)=f^{N}(x)
$$

(by convention $f^{0}(x)=x$ ). Take $N=\infty$ if

$$
f^{m}(x) \neq f^{n}(x)
$$

for all integers $0 \leq m<n$.

Definition 2 (see [3]). The function $f: A \rightarrow A$ is called acyclic, if for each element $x \in A$ there is an integer $0 \leq N=N(x) \leq \infty$ such that $f$ takes $N=N(x)$ steps on $x$.

A partially ordered unary algebra $\left(A, f, \leq_{r}\right)$ is called acyclic, if $f: A \rightarrow A$ is acyclic. It is easy to see that any linearly ordered unary algebra is acyclic. If ( $A, f, \leq r$ ) is acyclic, then $r_{f}$ is an $f$-compatible partial order on $A$.

Lemma 1 (see [2]). Let $\left(A, f, \leq_{r}\right)$ be an acyclic partially ordered unary algebra. If $a, b \in A$ and $a \leq_{r} f(a)$ or $f(a) \leq_{r} a$, then $(a, b) \in r_{f}$ implies that $a=b$ or $f^{m}(a) \leq r f^{m}(b)$ and $f^{m}(a) \neq f^{m}(b)$ for some integer $m \geq 0$.

\section{THE ACYCLIC PARTIALLY ORDERED UNARY ALGEBRA $(A, f, \hat{f})$}

Let

$$
\langle x\rangle_{f}=\left\{x, f(x), f^{2}(x), \ldots\right\}
$$

denote the $f$-orbit of $x$ and define the following reflexive and transitive relation $\hat{f} \subseteq$ $A \times A$ as follows:

$$
x \hat{f} y \quad \Leftrightarrow \quad\langle x\rangle_{f} \subseteq\langle y\rangle_{f} .
$$


Clearly, $\langle x\rangle_{f} \subseteq\langle y\rangle_{f}$ if and only if $x \in\langle y\rangle_{f}$, i.e. we can find an integer $k \geq 0$ such that $x=f^{k}(y)$.

Proposition 1. If $(A, f)$ is a unary algebra, then the following are equivalent.

(1) $f$ is an acyclic function.

(2) $\hat{f}$ is antisymmetric.

Proof. $(1) \Rightarrow(2)$ : Suppose that $x \hat{f} y$ and $y \hat{f} x$ for $x, y \in A$. Then $\langle x\rangle_{f}=\langle y\rangle_{f}$ imply that $x=f^{m}(y)$ and $y=f^{n}(x)$ for some $m \geq 0$ and $n \geq 0$. Thus

$$
x=f^{m}\left(f^{n}(x)\right)=f^{m+n}(x),
$$

whence

$$
x=f(x)=\ldots=f^{n}(x)=\ldots=f^{n+m}(x)
$$

follows from the acyclic property of $f$. Obviously,

$$
x=f^{n}(x)=y .
$$

(2) $\Rightarrow(1)$ : Suppose that $f^{m}(x)=f^{n}(x)$ for some integers $0 \leq m<n$. Clearly, $\left\langle f^{m+1}(x)\right\rangle_{f} \subseteq\left\langle f^{m}(x)\right\rangle_{f}$ and $\left\langle f^{m}(x)\right\rangle_{f} \subseteq\left\langle f^{m+1}(x)\right\rangle_{f}$ is a consequence of

$$
f^{m}(x)=f^{n-m-1}\left(f^{m+1}(x)\right) .
$$

Thus we have

$$
f^{m}(x) \hat{f} f^{m+1}(x) \quad \text { and } \quad f^{m+1}(x) \hat{f} f^{m}(x) .
$$

Since $\hat{f}$ is antisymmetric, we get $f^{m}(x)=f^{m+1}(x)$ and

$$
f^{m}(x)=f^{m+1}(x)=\ldots=f^{n}(x) .
$$

Proposition 2. Let $(A, f)$ be an acyclic unary algebra. Then the partial order $\hat{f}$ is $f$-compatible.

Proof. If the function $f$ is acyclic, then $\hat{f}$ is a partial order by Proposition 1 . If $x \hat{f} y$ for $x, y \in A$, then we can find an integer $m \geq 0$ such that

$$
x=f^{m}(y),
$$

whence $f(x)=f^{m}(f(y))$ and $f(x) \hat{f} f(y)$ follows.

\section{THE INTERSECTION}

Proposition 3. If $(A, f)$ is an acyclic unary algebra and $\langle x\rangle_{f} \cap\langle y\rangle_{f} \neq \varnothing$ for $x, y \in A$, then there exists a unique $z \in A$ such that

$$
\langle x\rangle_{f} \cap\langle y\rangle_{f}=\langle z\rangle_{f} .
$$

This element $z=x \Delta y$ is called the $f$-intersection of $x$ and $y$. 
Proof. Since $\langle x\rangle_{f} \cap\langle y\rangle_{f} \neq \varnothing$, we can define an integer $n$ as follows:

$$
n=\min \left\{k \geq 0 \mid f^{k}(x) \in\langle y\rangle_{f}\right\} .
$$

We claim that $\langle x\rangle_{f} \cap\langle y\rangle_{f}=\langle z\rangle_{f}$ for $z=f^{n}(x)$. Obviously,

$$
\langle z\rangle_{f} \subseteq\langle x\rangle_{f} \cap\langle y\rangle_{f} .
$$

On the other hand if $u \in\langle x\rangle_{f} \cap\langle y\rangle_{f}$ then $u=f^{k}(x) \in\langle y\rangle_{f}$ for some $k \geq 0$. Thus $k \geq n$ and $u=f^{k-n}\left(f^{n}(x)\right)=f^{k-n}(z) \in\langle z\rangle_{f}$. The fact that there is only one $z \in A$ with

$$
\langle x\rangle_{f} \cap\langle y\rangle_{f}=\langle z\rangle_{f}
$$

is a consequence of Proposition 1.

Definition 3. Let $(A, f)$ be an acyclic unary algebra. If $\langle x\rangle_{f} \cap\langle y\rangle_{f} \neq \varnothing$ for $x, y \in A$, then define the distance of $x$ and $y$ as follows:

$$
\delta(x, y)=\left|\left(\langle x\rangle_{f} \backslash\langle y\rangle_{f}\right) \cup\left(\langle y\rangle_{f} \backslash\langle x\rangle_{f}\right)\right|=\left|\langle x\rangle_{f} \backslash\langle y\rangle_{f}\right|+\left|\langle y\rangle_{f} \backslash\langle x\rangle_{f}\right| .
$$

We note that

$$
\delta(x, y)=\left|\langle x\rangle_{f} \backslash\langle x \Delta y\rangle_{f}\right|+\left|\langle y\rangle_{f} \backslash\langle x \Delta y\rangle_{f}\right| .
$$

immediately follows from

$$
\langle x\rangle_{f} \backslash\langle y\rangle_{f}=\langle x\rangle_{f} \backslash\left(\langle x\rangle_{f} \cap\langle y\rangle_{f}\right)=\langle x\rangle_{f} \backslash\langle x \Delta y\rangle_{f}
$$

and

$$
\langle y\rangle_{f} \backslash\langle x\rangle_{f}=\langle y\rangle_{f} \backslash\langle x \Delta y\rangle_{f} .
$$

Proposition 4. If $(A, f)$ is an acyclic unary algebra and $\langle x\rangle_{f} \cap\langle y\rangle_{f} \neq \varnothing$ for $x, y \in A$, then

$$
\left|\langle x\rangle_{f} \backslash\langle y\rangle_{f}\right|=\left|\langle x\rangle_{f} \backslash\langle x \Delta y\rangle_{f}\right|=\min \left\{k \geq 0 \mid f^{k}(x) \in\langle y\rangle_{f}\right\} \leq N(x),
$$

where the integer $N(x)$ is the number of $f$-steps on $x$.

Proof. If $n=\min \left\{k \geq 0 \mid f^{k}(x) \in\langle y\rangle_{f}\right\}$, then we have

$$
x \triangle y=f^{n}(x)
$$

(see the proof of Proposition 3). We distinguish two cases.

Case 1 If $N(x)=\infty$, then $n<N(x)$ and

$$
\langle x\rangle_{f} \backslash\left\langle f^{n}(x)\right\rangle_{f}=\left\{f^{k}(x) \mid k \geq 0\right\} \backslash\left\{f^{k}(x) \mid k \geq n\right\} .
$$

Thus

$$
\left|\langle x\rangle_{f} \backslash\left\langle f^{n}(x)\right\rangle_{f}\right|=\left|\left\{x, f(x), \ldots, f^{n-1}(x)\right\}\right|=n .
$$

Case 2 If $N=N(x)<\infty$ and $N<n$, then

$$
\langle x\rangle_{f} \cap\langle y\rangle_{f}=\left\{x, f(x), \ldots, f^{N}(x)\right\} \cap\langle y\rangle_{f}=\varnothing,
$$


a contradiction. Thus $n \leq N$ and

$$
\langle x\rangle_{f} \backslash\left\langle f^{n}(x)\right\rangle_{f}=\left\{x, f(x), \ldots, f^{N}(x)\right\} \backslash\left\{f^{n}(x), \ldots, f^{N}(x)\right\},
$$

i.e.

$$
\left|\langle x\rangle_{f} \backslash\left\langle f^{n}(x)\right\rangle_{f}\right|=\left|\left\{x, f(x), \ldots, f^{n-1}(x)\right\}\right|=n .
$$

The next theorem gives a complete description of the intersection of the $f$-compatible linear extensions of the partial order $\hat{f}$.

Theorem 1. If $(A, f)$ is an acyclic unary algebra and $x, y \in A$, then:

$$
(x, y) \in(\hat{f})_{f} \Leftrightarrow x=y \text {, or }\langle x\rangle_{f} \cap\langle y\rangle_{f} \neq \varnothing \text { and } \delta(x, x \Delta y)<\delta(y, x \Delta y) .
$$

Proof. Since $\langle f(x)\rangle_{f} \subseteq\langle x\rangle_{f}$ (i.e. $\left.f(x) \leq \hat{f} x\right)$ for all $x \in A$, the application of Lemma 1 gives that

$(\hat{f})_{f}=\left\{(x, y) \in A \times A \mid(\exists m) 0 \leq m, f^{m}(x) \hat{f} f^{m}(y), f^{m}(x) \neq f^{m}(y)\right\} \cup\{(x, x) \mid x \in A\}$.

If $(x, y) \in(\hat{f})_{f}$ and $x \neq y$, then $\left\langle f^{m}(x)\right\rangle_{f} \subseteq\left\langle f^{m}(y)\right\rangle_{f}$ and $f^{m}(x) \neq f^{m}(y)$ for some integer $m \geq 0$. Suppose that

$$
k=\delta(x, x \Delta y) \geq \delta(y, x \Delta y)=l .
$$

Since $f^{m}(x) \in\langle y\rangle_{f}$, we have $m \geq k$ (see Proposition 4). Now

$$
f^{m}(x)=f^{m-k}\left(f^{k}(x)\right)=f^{m-k}(x \triangle y)
$$

and

imply that

$$
f^{m}(y)=f^{m-l}\left(f^{l}(y)\right)=f^{m-l}(x \triangle y)
$$

$$
f^{m}(y)=f^{k-l}\left(f^{m}(x)\right) .
$$

It follows that $\left\langle f^{m}(y)\right\rangle_{f} \subseteq\left\langle f^{m}(x)\right\rangle_{f}$. The antisymmetric property of $\hat{f}$ gives that $f^{m}(x)=f^{m}(y)$, a contradiction.

If $\langle x\rangle_{f} \cap\langle y\rangle_{f} \neq \varnothing$ and

$$
m=\delta(x, x \Delta y)<\delta(y, x \triangle y)=l,
$$

then

$$
f^{m}(x)=x \Delta y=f^{l}(y)=f^{l-m}\left(f^{m}(y)\right)
$$

ensures that $\left\langle f^{m}(x)\right\rangle_{f} \subseteq\left\langle f^{m}(y)\right\rangle_{f}$. On the other hand, using Proposition 4,

$$
m<l \leq N(y)
$$

implies that

$$
f^{m}(y) \neq f^{l}(y)=f^{m}(x) .
$$

Thus we have $(x, y) \in(\hat{f})_{f}$. 


\section{ACKNOWLEDGEMENT}

This research was carried out as part of the TAMOP- 4.2.1.B-10/2/KONV-20100001 project with support from the European Union, co-financed by the European Social Fund.

\section{REFERENCES}

[1] S. Foldes and J. Szigeti, "Maximal compatible extensions of partial orders," J. Aust. Math. Soc., vol. 81, no. 2, pp. 245-252, 2006.

[2] J. Szigeti, "On the intersection of monotonicity preserving linear extensions," Acta Math. Hung., vol. 55, no. 1-2, pp. 161-163, 1990.

[3] J. Szigeti and B. Nagy, "Linear extensions of partial orders preserving monotonicity," Order, vol. 4, no. 1-3, pp. 31-35, 1987.

[4] S. Szilágyi, "A characterization and the intersection of the maximal compatible extensions of a partial order," Order, vol. 25, no. 4, pp. 321-333, 2008.

[5] E. Szpilrajn, “Sur l'extension de l'ordre partiel," Fundamenta, vol. 16, pp. 386-389, 1930.

Author's address

\section{Szilvia Szilágyi}

University of Miskolc, Institute of Mathematics, Miskolc-Egyetemváros, 3515 Miskolc, Hungary

E-mail address: matsziszouni-miskolc.hu 